\title{
Adherencia a la dieta mediterránea y su influencia en la condición física en niños de 3 a 6 años \\ Adherence to the mediterranean diet and how it can influence in the physical fitness of 3 to 6 -year-old children \\ Gema Díaz-Q uesada, Inés M uñoz-Galiano, GemaTorres-Luque \\ Universidad de Jaén (España)
}

\begin{abstract}
Resumen. Introducción: La primera etapa escolar podría servir para enseñar hábitos saludables a nivel de alimentación y actividad física(AF). 0 bjetivo: Determinar las diferencias en condición física (CF) en función del grado de adherenciaaladieta mediterránea (DM ). M etodología: Se seleccionaron 97 escolares de entre 3 y 6 años, a los que se les eval uó la CF, a través de la batería de FIT ness en PREescolares (PREFIT); y se determinó su nivel de adherencia a la DM por medio del cuestionario KIDMED. Resultados: Los sujetos mostraron valores de media y alta adherencia a la DM, no existiendo casos de baja adherencia. Existen pocas diferencias, aunque el grupo de media adherencia tiene mejores valores en salto de longitud, equilibrio y velocidad. Conclusión: En el primer periodo infantil aún se observan pocas diferencias entrelos sujetos con media y alta adherencia a la DM. Es necesario seguir profundizando en la influencia de factores relacionados con la salud, en poblaciones infantiles para poder proponer en el futuro propuestas de mejora.
\end{abstract}

Palabras clave: educación, infantil, salud, actividad física.

\begin{abstract}
Background: The first stage of school could serve to teach healthy habits at the level of food and physical activity. 0 bjective: To determine the differences in physical fitness based on the degree of adherence to the mediterranean diet. M ethods: 97 schoolchildren between 3 and 6 years old were selected to whom the physical fitness was evaluated throught the FIT ness battery in PREschool (PREFIT); and their leve lof adherence to the mediterranean diet was determined by means of the KIDMED questionnaire. Results: The subjects cases mean values and high adherence to DM, there are no cases of low adherence. There are few differences, although the group of adhesion means has better values in long jump, balance and speed. Conclusion: In the first infant period there are still few differences between subjects with medium and high adherence to DM. It is necessary to continue to deepen the influence of health-related factors in children's populations in order to propose proposals for improvement in the future.
\end{abstract}

Key words: education, preschool, health, physical activity.

\section{Introducción}

El primer período escolar es muy significativo, ya que todo lo que se desarrolle en él, servirá para las siguientes etapas de la vida. En dichas etapas el sujeto se desarrollará a nivel físico, social y cognitivo (Abad, Ca ñada \& Maraflores, 2016). Este tiempo, es crucial para empezar a desarrollar una buena condición física (CF), y el entorno escolar es un lugar idóneo para alcanzar estilos de vida activos y saludables. A su vez, esto ayuda rá a que el profesorado cumpla con los objetivos normativos del currículum de educación infantil, como son el desarrollo corporal y el movimiento (Kryst et al., 2016; Gil-Espinosa, Romance \& Nielsen, 2018; Arufe Giráldez, 2019).

Para la evaluación de la CF existen actualmente di-

Fecha recepción: 13-03-20. Fecha de aceptación: 18-01-21 Inés Muñoz-Galiano

imunoz@ujaen.es ferentes baterías de test que se emplean en relación a la edad del sujeto. Tal como indica Ayán-Pérez (2013) la variedad es amplia y los profesionales deben estar formados y capacitados para determinar o seleccionar las pruebas en relación a sus recursos, validez, fiabilidad, etc. Entre las baterías existentes en la literatura científica, una de las más extendidas es la batería APLHA (Ruiz, et al., 2011) para sujetos de entre 6 y 12 años. En cuanto a edades tempranas entre los 3 a 6 años, existen distintas propuestas de acuerdo al creciente interés por el tema, como, por ejemplo: Movement Assessment Battery for Children-2 (Veldhuizen, Rivard \& Cairney, 2017), batería PREFIT (Cadenas-Sánchez, et al., 2016; O rtega, et al., 2015), el Test of Gross Motor Development TGMD-3 (M aeng, Webster \& Ulrich, 2016) entre otras (Latorre-Román, Fernández-Sánchez, M oriana Coronas \& García-Pinillos, 2016). Con los resultados que se obtienen de dichas pruebas se pueden observar y analizar los niveles de CF que tienen los sujetos. De este modo se determina si el sujeto se encuentra en un nivel adecuado o si, por el contrario, el 
sujeto comienza atener problemas relacionados con este ámbito (M artínez-Téllez, et al., 2016).

En este sentido, la ingesta al imenticia tiene un papel importante. En el contexto mediterráneo, se ha comprobado cómo la dieta mediterránea (DM) es una de las propuestas más saludables que existen (Kain, Leyton, Concha, W isstaub, Lobos, Bustos \& Vio, 2012). La misma, está basada en el consumo diario de alimentos de origen vegetal (cereales, legumbres, frutas, verduras, frutos secos, pan y aceite de oliva); el consumo modera do de pescados y mariscos; el consumo bajo-moderado de productos lácteos y huevos; y el consumo bajo de carnes rojas, alcohol y dulces. A lo que habría que aña dir la importancia de la realización diaria de actividad física(AF) (D omenech-A sensi, Sánchez-M artínez \& RosBerruezo, 2014).

Se ha demostrado que el consumo de la DM y la realización deAF, previene diversas enfermedades como la diabetes, la obesidad, enfermedades neurodegenerativas, cardiovasculares, cerebrovasculares e incluso el cáncer (García-Cabrera, HerreraFernández, Rodríguez-Hernández, Nissensohn, RománVinas \& Serra-Majem, 2015; Grosso, et al., 2013). A pesar de ello, estudios recientes muestran que, en los últimos años, ha disminuido el tiempo de realización de AF (Andersen, Mota \& Di Pietro, 2016; Tremblay et al., 2017), así como el consumo de una DM, incrementándose el consumo de comida rápida, comida fuera del hogar 0 alimentos procesados (G rao-Cruces, Nuviala, Fernández-Martínez, Porcel-Gálverz, MoralGarcía \& Martínez-López., 2013; Sahingoz \& Sanlier, 2011).

El principal lugar donde se debe promover en el al umnado la realización de AF y el desarrollo de hábitos alimenticos saludables es en el hogar. Las familias deben brindar tiempo y espacios para que los niños/ as puedan jugar y moverse, además de ello el hogar también debe proporcionar una buena DM, dando acceso y disponibilidad para consumir frutas y verduras, y evitando comidas rápidas o bebidas endulzadas ( 0 stbye, Malhotra, Stroo, Lovelady, Brouwer, Zucker \& Fuemmeler, 2013). Diferentes estudios han analizado que, si se realizan programas de AF combinados con el consumo diario de laDM , problemascomo el sobrepeso y la obesidad empezarían a disminuir, por lo que la CF de los individuos mejoraría (Kain, et al., 2012; Nava, Pérez, Herrera \& Hernández, 2011).

Como se viene constatando, la CF es claramente un factor indicativo de salud, al igual que la DM es un pa trón nutritivo para la misma, por lo que resulta crucial unir ambos conceptos para tener una mayor calidad de vida y reducir problemas de sobrepeso, obesidad, y prevenir enfermedades (Lavie, M cAuley, Church, Milani \& Blair, 2014; G aesser, Tucker, Jarrett \& Angadi, 2015; Serra-Majem, Ribas, N go, O rtega, García, PérezRodrigo \& Aranceta, 2019).

Desde esta perspectiva, se precisan más estudios en este sentido en edades tempranas con la intención de prevenir y crear hábitos saludables. La mayoría se centran en población adolescente o escolares de primaria, siendo muy escasa la información en población infantil. Por lo tanto, el objetivo de este estudio es determinar las diferencias existentes en la condición física según el grado de adherencia a la dieta mediterránea de escola res entre 3 a 6 años.

\section{Material y método}

\section{Muestra}

Se seleccionaron un total de 97 alumnos/ as de segundo ciclo de Educación Infantil (4,21 1 , 78 años), de los cuales $n=23(23,7 \%)$ pertenecen a primer curso de infantil ( 3 años), $n=36(37,1 \%$ ) a segundo curso (4 años) y $n=38(39,2 \%)$ al tercer curso (5 años). Del total de la muestra $n=52(53,6 \%)$ fueron niños y $n=45(46,4 \%)$ fueron niñas. El alumnado pertenece a dos centros educativos de entornos rurales de la provincia de Jaén.

Se informó tanto a los centros educativos como a las familias de los objetivos del estudio, donde los progenitores firmaron un consentimiento para participar. El estudio estuvo aprobado por el comité de ética de la institución local.

\section{Procedimiento}

La evaluación de la CF se llevó a cabo en los centros educativos, en una sala habilitada para tal efecto, donde se realizaron los test propuestos en la batería PREFIT (O rtega, et al. , 2015; Cadenas-Sánchez, et al., 2016).

\section{Valoración antropométrica}

Lavaloración antropométricase determinó mediante la medición de la masa, la talla y el perímetro de la cintura $(\mathrm{cm})$, en un aula habilitada para tal efecto. La medición de la masa se realizó dos veces con los sujetos descalzos y con ropa ligera. Los sujetos se colocaron de pie sobre la báscula eléctrica modelo SECA (SECA LTD., Germany) y se registró el resultado en kilogra mos. Para la talla, se midieron dos veces a cada uno de los sujetos descalzos, de pie, con los talones, glúteos y espalda en contacto con la pared. Con el metro abierto 
colocado en la pared con cinta aislante, se registró el resultado en centímetros. El perímetro de la cintura se midió también en dos ocasiones utilizando la cinta métrica inextensible milimetrada de fibra de vidrio Holtain y se registró el resultado en centímetros. El Índice de Masa Corporal (IMC) se calculó con la fórmula masa en kilogramos dividido por el cuadrado de la talla en metros $\left(\mathrm{kg} / \mathrm{m}^{2}\right)$. El resultado será más favorable cuanto más cercano se encuentre entre los valores 18,5 y 25.

\section{Fuerza isométrica manual}

Cada sujeto se situó de pie, erguido, con las piernas ligeramente separadas y los brazos extendidos verticalmente a lo largo del tronco, pero sin tocar ninguna parte de éste. Con esta posición el sujeto debía presionar todo lo que pudiese sobre el Dinamómetro M anual Test 320, flexionando los dedos de la mano. En el momento en que conseguía su grado máximo de flexión se registraba la marca en kilogramos. Se registraron dos intentos con cada mano, con un minuto de recuperación, donde se real izaba primero con la mano derecha y después con la izquierda. El resultado será más favorable cuanto mayor sea la marca.

\section{Evaluación del salto horizontal}

Los sujetos se colocaron de pie tras la línea de salto marcada con cinta aislante. Tenían la separación de los pies igual a la anchura de sus hombros y a la derecha se encontraba el metro abierto colocado con cinta aislante. Desde esa posición doblaban las rodillas con los brazos delante del cuerpo y paralelos al suelo, balanceaban los brazos, empujaban con fuerza y saltaban lo más lejos posible. Cuando sus pies tocaban el suelo debían quedarse quietos. Se realizó 3 veces, el descanso entre repeticiones fue de un minuto y la distancia se registraba en centímetros. El resultado será más favorable cuanta más distancia recorra.

Evaluación del equilibrio con una pierna

El sujeto se colocó de manera estática sobre el suelo y comenzaba flexionando la pierna izquierda, se registraron dos intentos con cada pierna, alternando primero derecha, después izquierda, y se anotaron los segundos que conseguían mantenerse en esa posición. Esta prueba evaluaba el equilibrio estático. El resultado se registró en segundos con un decimal. El resultado será más favorable cuanto más segundos aguante.

Evaluación del gateo

Se realizó en un aula habilitada para tal efecto, el sujeto debía colocarse en cuadrupedia, ir lo más rápido posible hasta un obstáculo colocado a 5 metros de distancia, rodearlo y volver hasta la salida. El resultado se registraba en segundos con un decimal. Esta prueba no está dentro de la batería PREFIT, pero ha sido empleada por otros estudios para muestras de estas edades para valorar lacoordinación dinámica general (Torres-Luque, Hernández-García, O rtega-Toro \& Nikolaidis, 2018). El resultado será más favorable cuantos menos segundos tarde.

Evaluación de la velocidad agilidad $4 \times 10 \mathrm{~m}$

Se llevó a cabo en una pista al aire libre, se marca ron dos líneas paralelas en el suelo con tiza a 10 metros de distancia colocándose un adulto en cada extremo. Cuando se indicaba la salida el sujeto debía correr lo más rápido posible hasta la otra línea, chocaba la mano del adulto 1 y volvía a la línea de sal ida, cruzando ambas líneas con los dos pies y chocando la mano del adulto 2 . Seguidamente, corría lo más rápido posible a la línea opuesta, chocaba la mano al adulto 1 y volvía corriendo a la línea de salida inicial donde chocaba la mano del adulto 2. El tiempo se registraba en segundos con un decimal. El resultado será más favorable cuantos menos segundos tarde.

Test de Course $N$ avette

Se realizó en una pista al aire libre y se marcaron dos líneas paralelas en el suelo con tizas a 20 metros de distancia. Consistía en una prueba de intensidad progresiva y máxima donde se debía recorrer una distancia de 20 metros siguiendo el ritmo de la grabación que tenía un adulto, queiba aumentando de forma progresivacada minuto, comenzaba con una velocidad de $6,5 \mathrm{~km} / \mathrm{h}, \mathrm{y}$ se incrementaba en $0,5 \mathrm{~km} / \mathrm{h} / \mathrm{min}$ (1 minuto es igual a 1 palier/ estadio). La prueba se hacía en grupos de 6 sujetos que corrían a la vez junto con un adulto desde una línea hasta la otra, cuando un sujeto no llegaba por segunda vez consecutiva a una de las líneas con la señal de audio o no podía continuar por la fatiga, se iba con el otro adulto que estaba registrando las marcas. Para el resultado, el adulto apuntaba el número de vueltas tota les que había hecho el individuo, observaba la tabla con los datos y anotaba el número de estadio, velocidad y número de vueltas. El resultado serámásfavorable cuanto mayor sea el número de estadío en el que se encuentre.

Nivel de adherencia a la dieta mediterránea (KIDMED) Se empleó el cuestionario de adherencia a la DM, 
KIDM ED (Serra-M ajem, et al. , 2004). Este instrumento consta de 16 preguntas dicotómicas que deben de responderse de manera afirmativa/ negativa (sí/ no). Las respuestas afirmativas en las preguntas que representan un aspecto positivo en relación con la DM (12 preguntas) suman un punto, mientras que las respuestas afirmativas en las preguntas que representan una connotación negativa respecto a la DM (cuatro preguntas) restan un punto. Las respuestas negativas no puntúan. Lapuntuación total obtenidadalugar al índiceKIDMED, que se clasifica en tres categorías: a) De 8 a 12: DM óptima (alto grado de adherencia); b) De 4 a 7 : N ecesidad de mejorar el patrón al imentario para adecuarlo al modelo mediterráneo (grado de adherencia medio); y, c) De 0 a 3: Dieta de muy baja calidad (bajo grado de adherencia a la DM). El cuestionario fue completado por los progenitores de los sujetos.

\section{Análisis estadístico}

Se empleó el paquete estadístico SPSS versión 21.0 paraW indows (IBM Software Group, Chicago, Illinois, Estados U nidos). Se realizó un análisis descriptivo de los datos mostrándolos como media y desviación típica en formato tabla. Se empleó el test de normalidad Kolmogorov-Smirnov, que confirmó una distribución normal. Se utilizó el t-test para muestras independientes Student's para la comparación de la CF en relación al nivel de adherencia a la DM (media versus alta). También se utilizó el t-test para muestras independientes Student's para la comparación de la CF en función de la adherencia a la DM (media versus alta) en relación al género (chicos versus chicas). Dentro del análisis estadístico t-test para muestras independientes Student's se ha llevado a cabo un análisis sobre el tamaño del efecto (Cohen, 1988), dicho análisis se divide en 3 categorías: 0,20 valor bajo, 0,50 valor medio, 0,80 valor alto. La significación se fijó en $p<$ 0.05 para las diferentes pruebas.

\section{Resultados}

\section{Diferencias en la condición física en función de adherencia a la dieta mediterránea en relación al género}

Los resultados del nivel de adherencia a la DM indica que no hubo ningún sujeto con una baja adherencia a la DM, por lo que la comparativa se realizó entre los grupos de media y alta adherencia.

En laTabla 1 se muestran los resultados de condición física respecto al nivel de adherencia a la DM.

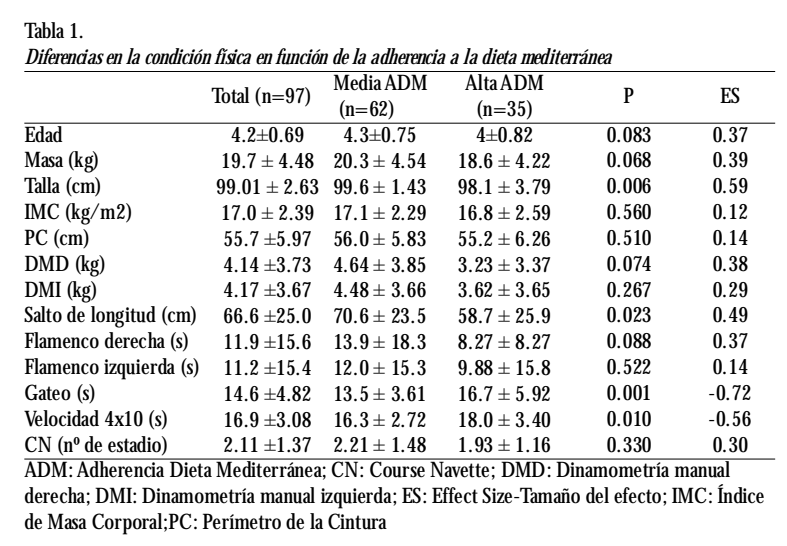

Según muestran los resultados, se observan diferencias estadísticamente significativas en talla, salto de longitud, prueba de gateo y velocidad $4 \times 10$, con mejores valores en el grupo de media adherencia a la dieta mediterránea, frente a los que tienen una alta adherencia $(p<0.05)$. Siendo a su vez el tamaño del efecto de las mismas de valor medio.

En laTabla 2 se muestran los resultados de condición física respecto al nivel de adherencia a la DM en rela ción al género.

Tabla 2.

Diferencias en la condición física en función de la adherencia a la dieta mediterránea en relación al género Chicos $(n=55) \quad \operatorname{Chicas~}(n=42)$

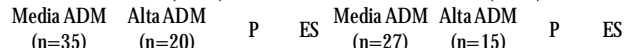

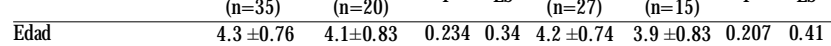
$\begin{array}{lcccccccc}\text { Edad } & 4.3 \pm 0.76 & 4.1 \pm 0.83 & 0.234 & 0.34 & 4.2 \pm 0.74 & 3.9 \pm 0.83 & 0.207 & 0.41 \\ \text { M asa }(\mathrm{kg}) & 20.5 \pm 4.20 & 19.1 \pm 4.09 & 0.245 & 0.33 & 20.1 \pm 5.01 & 17.9 \pm 4.42 & 0.160 & 0.46\end{array}$ Talla (cm) $\quad 99.8 \pm 1.14 \quad 99.0 \pm 2.390 .0950 .4899 .3 \pm 1.7296 .9 \pm 4.940 .026 \quad 0.75$ $\begin{array}{llllllll}\text { IMC }(\mathrm{kg} / \mathrm{m} 2) & 17.0 \pm 2.42 & 16.7 \pm 2.85 & 0.701 & 0.19 & 17.1 \pm 2.1516 .8 \pm 2.29 & 0.655 & 0.14\end{array}$

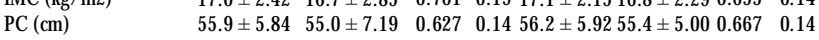
DMD (kg) $\quad 5.00 \pm 3.613 .88 \pm 3.08 \quad 0.249 \quad 0.334 .19 \pm 4.172 .38 \pm 3.650 .168 \quad 0.45$ DMI (kg) $\quad 4.46 \pm 3.63 \quad 4.10 \pm 3.24 \quad 0.712 \quad 0.104 .51 \pm 3.782 .98 \pm 4.170 .233 \quad 0.39$

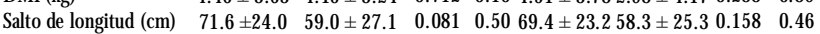
$\begin{array}{llllllll}\text { Flamenco derecha }(\mathrm{s}) & 14.2 \pm 21.6 & 6.65 \pm 9.18 & 0.281 & 0.31 & 13.5 \pm 13.37 .76 \pm 7.16 & 0.126 & 0.50\end{array}$

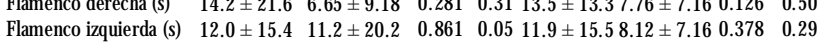

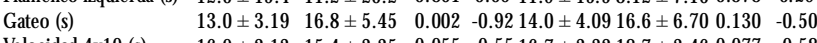
$\begin{array}{lllllllll}\text { Velocidad } 4 \times 10(s) & 16.0 \pm 2.13 & 15.4 \pm 3.35 & 0.055 & -0.55 & 16.7 \pm 3.3218 .7 \pm 3.46 & 0.077 & -0.58\end{array}$

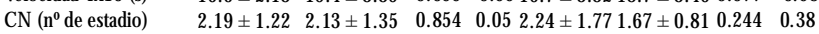
ADM: Adherencia Dieta M editer ránea; CN: Course Navette; DM D: Dinamometría manual derecha; DMI: Dinamometría manual izquierda; ES: Effect Size - Tamaño del efecto; IMC: Índice de Masa Corporal; PC Perímetro de lacintura

Según muestran los resultados, se observan diferencias estadísticamente significativas en chicos en el gateo $(p<0.05)$, y en chicas en la talla $(p<0.05)$. Siendo a su vez el tamaño del efecto en el gateo de un valor muy bajo y en la talla un valor cercano a alto.

\section{Discusión}

Este trabajo observa las diferencias en la CF respecto al nivel de adherencia a la DM en edades tempranas. Los principales hallazgos de este estudio muestran como en la población seleccionada, no existe ningún caso de baja adherencia a la dieta mediterránea. Además, las diferencias entre media y alta adherencia a la dieta mediterránea respecto a la condición física se muestran en diversas variables como las relacionadas con velocidad y fuerza, y que respecto al género las variables cambian. 
En un inicio, destaca como no existen sujetos con baja adherencia a la dieta mediterránea. Esto es un aspecto relevante, ya que, en estudios con muestras con una edad mayor, hay una tendencia a la presencia de este grupo (Cömert, Cerkez, Tekin, Aydogan \& Essiz, 2015; Bibiloni, Pons\&Tur, 2015; Barja-Fernández, Pino, Portela \& Leis, 2019). Los motivos no se han analizado en el presente trabajo, aunque se ha evidenciado que según avanza la edad y los sujetos tienen mayor autonomía, suelen desarrollar hábitosmenos saludables en cuanto a alimentación (Grosso, et al., 2013; Grao-Cruces, et al., 2013). No obstante, parece interesante destacar que, en la muestra seleccionada, no exista ningún caso de baja adherencia.

Respecto a los resultados concernientes a la CF respecto al nivel de adherencia a la DM, los valores halla dos en ambos grupos están acorde a lo mostrado en otras investigaciones con sujetos de estas edades (Latorre- Román, et al., 2016; Torres-Luque, et al., 2018). Sin embargo, Ilama la atención como la tendencia es a obtener mejores valores en el grupo con una media adherencia a la dieta mediterránea (tabla 1). Las pruebas relacionadas con fuerza, como son el test de dinamometría manual y salto horizontal, son mayores en el grupo de media adherencia, siendo significativa en la segunda. No se ha encontrado información que indique las posibles diferencias en este aspecto relacionado con la DM, aunque si se sabe que la fuerza, tiende a tener valores constantes en la etapa de infantil (KingDowling, Proudfoot, Cairney \& Timmons, 2017), aunque se incrementa con la edad entre los 3 y 5 años (Torres-Luque, et al. , 2018). Se ha observado como el peso y la talla pueden influenciar en el resultado a estas eda des (Davis, Kang, Boswell, DuBose, Altman \& Binkley, 2008; M cK ay, et al., 2017) y, en la muestra sel eccionada el grupo de media adherencia tiene valores más altos en talla. Si bien es cierto, que ambos grupos, se encuentran por debajo de lo determinado por otros estudios respecto a estas edades (Wang, Wu \& Chang, 2013; Torres-Luque, et al., 2018).

En relación al tiempo evaluado por medio de la prueba de gateo y $4 \times 10 \mathrm{~m}$, obtiene resultados más elevados el grupo de alta adherencia a la DM $(p<0.05)$ (tabla 1$)$, pero eso no indica que sean mejores resultados, al contrario, son peores resultados, puesto que, a mayor tiempo de realización de la prueba, peores valores se obtienen. Basándose en los hallazgos de la literatura científica, parece el lugar de residencia puede influir, ya que los países desarrollados tienden a tener valores menos próximos a los encontrados en este estudio (Cadenas-
Sánchez, et al., 2014, 2016) y similares a los de este estudio y a otros tipos de poblaciones con niveles socioeconómicos más bajos (Wang et al., 2013), más que en el tipo de dieta. Se ha evidenciado como en países desarrollados, existe una mayor tendencia a la comida «basura», fundamental mente en la transición de niño a adolescente (Torres-Luque, et al., 2018).

Una de las limitaciones de este estudio es que no se ha analizado el nivel deAF y los hábitos nutricionales de las familias, que quizás podría haber ar rojado mayor información a los resultados (Nikolaidis, 2011). En lo referente a los resultados obtenidos a nivel cardiorrespiratorio, se observa que los sujetos en ambos grupos muestran resultados muy similares, pudiendo destacar diferencias solo en el número de estadios, donde en esta ocasión el grupo con baja adherencia a la DM obtiene valores más elevados.

Respecto a los resultados relacionados con el género, se puede observar que los valores tanto en media como en alta adherencia a la DM tienden a tener la misma tendencia tanto en chicos como en chicas. A pesar de no haber realizado la diferencia chicos versus chicas, se muestra una tendencia en parámetros de fuerza, así como de talla y masa mayores en chicos, coincidiendo con otros estudios (Wang et al., 2013; MartínezTéllez et al., 2016; Torres-Luque et al., 2018). A este respecto, la DM no parece tener influencia analizando el género de manera independiente.

En la evaluación de la velocidad, los chicos con media adherencia a la DM muestran tener mejores valores respecto a los de alta adherencia, con valores para ambos casos, dentro de lo determinado por otros investigadores (Wang et al., 2013; Andrade et al., 2014; Martínez-Téllez et al., 2016). Q uizás el dato que más pueda llamar la atención, es como existen valores muy equitativos en cuando al grado de adherencia a la DM, aspecto anal izado en otrosestudios (Ayuchu et al. , 2010; Grao-Cruces et al., 2013; Archero et al., 2018), aunque en este estudio no se muestra. En este sentido, sería conveniente aumentar el número de la muestra y analizar la influencia de la familia, pues los hábitos alimentarios en edad escolar vienen marcados por los progenitores (Sahingoz, \& Sanlier, 2011).

En síntesis, en este estudio, existe tendencia a una media y alta DM donde la media adherencia a la misma, ya ofrece mejores valores respecto a la CF, aspecto que permanece constante en relación al género. Dado que, se ha mostrado el nivel de dependencia parental en estas edades, es interesante seguir analizando parámetros que tengan relación con hábitos saludables 
desde edades tempranas, para poder generar propuestas de mejora desde la etapa de Educación Infantil.

\section{Conclusiones}

En el presente estudio no se muestran sujetos con una baja adherencia a la dieta mediterránea. En la comparación entre media y altaadherencia, se observa como el primer grupo muestra valores significativamente mejores en parámetros de fuerza y velocidad. La misma tendencia existe cuando se analizan de forma independiente por género, aunque no con diferencias destacables.

Es necesario seguir profundizando en la influencia de factores relacionados con la salud en poblaciones infantiles para poder proponer en el futuro propuestas de mejora.

\section{Referencias}

Abad, B., Cañada, D. \& M iraflores, E. (2016). Acividad física y salud de 3 a 6 años guía para docentes de educación infantil. Madrid: Ministerio de Sanidad, Servicios Sociales e lgualdad.

Andersen, L. B., Mota, J. \& Di Pietro, L. (2016). Update on the global pandemic of physical inactivity. The Lancet, 388, 12551256. doi:10.1016/ S0140-6736(16)30960-6

Andrade, S., 0 choazAvilés, A., Lachat, C., Escobar, P.,Verstræeten, R., Van Camp, J., ... Kolsteren, P. (2014). Physical fitness among urban and rural Ecuadorian adolescents and its association with blood lipids: a cross sectional study. BMC Pediatrics, 14(1), 106. doi:10.1186/ 1471-2431-14-106

Archero, F., Ricotti, R., Solito,A., Carrera, D., Civello, F., DiBella, R., Bellone, S. \& Prodam, F. (2018). Adherence to the $M$ editerranean Diet among School Children and Adolescents Living in Northern Italy and Unhealthy Food Behaviors Associated to 0 verweight. N utrients, 10(9), 1322. doi: 10.3390/ nu10091322

Arufe Giráldez, V. (2019). ¿Cómo debe ser el trabajo de Educación Física en Educación Infantil? (¿How should Physical Education work in Early Childhood Education be?). Retos, (37), 588-596. https:/ / doi.org/ 10.47197/ retos. v37i37.74177

Ayán-Pérez, C. (2013). Valoración de la condición física en el contexto delaeducación infantil: aplicaciones prácticas Apunts Educación Física y Deportes, (112), 52-62. doi:10.5672/ apunts.2014- 0983.es.(2013/2).112.04

Ayechu A. \& DuraT. (2010). Calidad de los hábitos alimentarios (adherenciaala dietamediterránea) en los alumnos de educa ción secundaria obligatoria. Anales del Sistema Sanitario de Navarra, 33(1), 35-42.

BarjaFernández, S. , Pino, M., Portela, I. \& Leis, R. (2019). Evalua ción de loshábitos de alimentación y actividad física en escolares gallegos. Nutrición Hospitalaria, 1, 26. doi: http:/ / dx. doi.org/ 10.20960/ nh. 02668
Bibiloni, M., Pons, A. \& Tur, J. A. (2015). Compliance with the Mediterranean Diet Q uality Index (KIDM ED) among Balearic Islands' Adolescents and Its Association with Socioeconomic, Anthropometric and Lifestyle Factors. Annals of Nutrition and M etabolism, 68(1), 42-50. doi:10.1159/000442302

Cadenas-Sánchez, C., Alcántara-Moral, F., Sánchez-Delgado, G., Mora-González, J., Martínez-Téllez, B., Herrador-Colmenero, M., ... O rtega, F. B. (2014). Assessment of cardiorespiratory fitness in preschool children: Adaptation of the 20 metres shuttle run test. Nutrición Hospitalaria, 30(6), 1333-1343. doi:10.3305/ nh. 2014.30.6.7859

Cadenas-Sánchez, C., M artínez-Téllez, B., Sánchez-Delgado, G., MoraGonzález, J., Castro-Pinero, J., Lof, M., ... O rtega, F. B. (2016). Assessing physical fitness in preschool children: Feasibility, reliability and practical recommendations for the PREFIT battery. Journal of Science and Medicinein Sport, 19(11), 910-915. doi:10.1016/ j.jsams.2016.02.003

Cohen, J. (1988). Statistical power analysis for the behavioral sciences (2a ed.). Hillsdale, N): Erlbaum. doi:10.4324/ 9780203771587

Cömert,T. K., Çerkez, M., G. Tekin,A. G.,Aydoðan, N. \& Esqiz, Ö. (2015). Compliance with Mediterranean Diet Quality Index (Kidmed) and Eating Patterns in School-age Children with Gaziantep,Turkey. American Journal of Food and N utrition,3(1):2833. doi: 10.12691/ ajfn-3-1-5

Davis, K. L., Kang, M., Boswell, B. B., DuBose, K. D.,Altman, S. R. $\&$ Binkley, H. M. (2008). Validity and reliability of the medicine ball throw for kindergarten children. The Journal of Strength and Conditioning Research, 22(6), 1958-1963.

Domenech-Asensi, G. , Sánchez-M artínez, A. \& Ros-Berruezo, G. (2014). Cross-sectional study to eval uatetheassociated factors with differences between city and districts secondary school students of the southeast of Spain (M urcia) for their adherence to the Mediterranean diet. N utrición H ospitalaria, 31(3), 13591365. doi:10.3305/ nh.2015.31.3.8306

Gaesser, G. A., Tucker, W. J., Jarrett, C. L. \& Angadi, S. S. (2015). Fitness versus Fatness W hich Influences Health and Mortality Risk theM ost? Current Sports MedianeReports, 14(4), 327-332. doi:10.1249/ jsr.0000000000000170

García-Cabrera, S., HerreraFernández, N., Rodríguez-Hernández, C., Nissensohn, M., Román-Vinas, B. \& Serra-Majem, L. (2015). KIDM ED test; prevalence of low adherence to the Mediterranean Diet in children and young; asystematicreview. Nutrición H ospitalaria, 32(6), 2390-2399. doi:10.3305/ nh. 2015.32.6.9828

Gil-Espinosa, F., Romance García, Ángel, \& Nielsen Rodríguez,A. (2018). Juego y actividad física como indicadores de calidad en Educación Infantil (Gamesand physical activity asindicators of quality in Early Childhood Education). Retos, (34), 252257. doi: $10.47197 /$ retos. V0i34.60391

Grao-Cruces, A., Nuviala, A., Fernández-Martínez, A., PorcelGálvez, A.M., Moral-García, J.E. \& Martínez- López, E. J. (2013). Adherencia a la dieta mediterránea en adolescentes rurales y urbanos del sur de España, satisfacción con la vida, antropometríay actividadesfísicasy sedentarias. Nutrición $\mathrm{H}$ os pitalaria, 28(4), 1129-1135. doi:10.3305/ 
nh. 2013.28.4.6486

Grosso, G., Marventano, S., Buscemi, S. , Scuderi, A., M atalone, M., Platania, A., ... Mistretta, A. (2013). Factors associated with adherence to the Mediterranean diet among adolescents living in Sicily, Southern Italy. Nutrients, 5(12), 4908-4923. doi:10.3390/ nu5124908

Kain, J., Leyton, B., Concha, F., Weisstaub, G., Lobos, L., Bustos, N. \&Vio, F. (2012). Evaluación deunaintervención en educación alimentaria y actividad física para prevenir obesidad infantil en escuelas públicas de Santiago de Chile. Archivos Latinoamericanos de Nutrición, 62 (1), 60-67. Recuperado de http:/ / w w w. s c i e l o. o r g. v e / scielo.php?script $=$ sci arttext $\&$ pid $=50004$ $06222012000100009 \& \operatorname{lng}=$ es $\&$ tlng=es

King-Dowling, S., Proudfoot, N. A., Carney, J. \&Timmons, B.W. (2017). Validity of field assessments to predict peak muscle power in preschoolers. Applied Physiology, Nutrition, and M etabolism, 42(8), 850-854, https:/ / doi. org/ 10.1139/ apnm-2016-0426

Kryst, L.,Woronkowicz, A., Jankowicz-Szymanska, A., Pociecha, M., Kowal, M., Sobiecki, .., . . . Zarow, R. (2016). Physical fitness of overweight and underweight preschool children from southern Poland. Anthropologischer Anzeiger, 73(2), 117124. doi:10.1127/ anthranz/2016/ 0561

Latorre-Román, P. A., Fernández-Sánchez, M., M oriana-Coronas, F. J. \& GarcíaPinillos, F. (2016). Design and validation of a cardiorespiratory capacity test for preschool children. South African Journal for Research in Sport Physical Education and Recreation, 38(2), 129-137. Recuperado de <Go to |S|>:/ / WOS:000383309300010

Lavie, C.J., M cAuley, P.A., Church,T. S., M ilani, R.V. \& Blair, S. N. (2014). $O$ besity and Cardiovascular Diseases. Journal of the American College of Cardiology, 63(14), 13451354. doi:10.1016/ j.jacc.2014.01.022

Maeng, H. J.,Webster, E. K. \& U Irich, D. A. (2016). Reliability for the Test of Gross M otor Development-Third Edition (TGMD-3). Research Quarterly for Exercise and Sport, 87, A38A38. Recuperado de $<\mathrm{Go}$ to $|\mathrm{S}|>: / \mid$ WOS:000383031300053

M artínez-Téllez, B., Sánchez-Delgado, G., Cadenas-Sánchez, C., M oraGonzález, J., Martín-M aillas, M., Lof, M., ... Ruiz, J. R. (2016). Health-related physical fitness is associated with total and central body fat in preschool children aged 3 to 5 years. Pediatric 0 besity, 11(6), 468-474. doi:10.1111/ ijpo. 12088

McKay, M. J., Baldwin, J. N., Ferreira, P., Simic, M., Vanicek, N., Burns, J. for the 1000 Norms Project Consortium (2017). Normativereferencevaluesfor strengthand flexibility of 1,000 children and adults. Neurology, 88(1), 36-43. doi: 10.1212/ W N L. 0000000000003466

Nava, M. C., Pérez,A., Herrera, H.A., yHernández, R.A. (2011). Hábitosalimentarios, actividad físicay su relación con el estado nutricional-antropométrico de preescolares. Revista chilena de nutrición, 38(3), 301-312. doi: 10.4067/ S071775182011000300006
Nikolaidis, P.T. (2011). Familial aggregation and maximal heritability of exercise participation: A cross-sectional study in schoolchildren and their nuclear families. Sci. Sports, 26(3) 157-165. doi: 10.1016/ j.scispo.2010.09.010

Ortega, F. B., Cadenas-Sánchez, C., Sánchez-Delgado, G., Mora González, J., Martínez-Téllez, B.,Artero, E. G.,... Ruiz,J. R. (2015). Systematic Review and Proposal of a Field-Based Physical Fitness-Test Battery in Preschool Children: The PREFIT Battery. SportsM ediane,45(4), 533-555. doi:10.1007/ S40279-014- 0281-8

O stbye, T., Malhotra, R., Stroo, M., Lovelady, C., Brouwer, R., Zucker, N., \& Fuemmeler, B. (2013). The effect of the home environment on physical activity and dietary intakein preschool children. International Journal of Obesity, 37, 1314-1321. doi:10.1038/ijo. 2013.76

Ruiz, J. R., España,V., Castro, J.,Artero, E. G., O rtega, F. B., Cuenca, M., ... Castillo, M. J., (2011). BđeríaALPHAFitness: test de campo para la eval uación de la condición física relacionada con la salud en niños y adolescentes. Nutrición Hospitalaria, 26(6), 1210-1214.

Sahingoz, S.A. \& Sanlier, N. (2011). Compliancewith M editerranean Diet Q uality Index (KIDMED) and nutrition knowledgelevels in adolescents. A case study fromTurkey. Appetite, 57(1), 272277. doi:10.1016/ j.appet.2011.05.307

Serra Majem, L., Ribas, L., N go, J., O rtega, R. M., García, A., Pérez-Rodrigo, C. \&Aranceta, J. (2004). Food, youth and the Mediterranean diet in Spain. Development of KIDMED, Mediterranean Diet Q uality Index in children and adolescents. Public Health Nutrition, 7(07), 931-935. doi:10.1079/ phn2004556

Serra-M ajem, L., Román-Viñas, B., Sanchez-Villegas, A., GuaschFerré, M., Corella, D. \& LaVecchia, C. (2019). Benefits of the Mediterranean diet: Epidemiological and molecular aspects. Molecular Aspects of Medicine, 67, 1-55. doi:10.1016/ j.mam.2019.06.001

Torres-Luque, G., Hernández-García, R., Ortega-Toro, E. \& Nikolaidis, P. (2018). The Effect of Place of Residence on Physical Fitness and Adherence to M editerranean Diet in 35-Year-O Id Girlsand Boys: U rban vs. Rural. N utrients, 10(12), 1855. doi:10.3390/ nu10121855

Tremblay, M. S., Aubert, S. , Barnes, J. D. , Saunders, T. J., Carson,V., Latimer-Cheung, A. E., Chastin, S. F. M., Altenburg, T. M., Chinapaw, M. J. M., \& on behalf of SBRN Terminology Consensus Proyect Participants. (2017). Sedentary Behavior Research N etwork (SBRN) - Terminology ConsensusProject processand outcome. International Journal of Behavioral Nuttrition and Physical Activity. 14, 75. doi:10.1186/ s12966-017-05258

Veldhuizen, S., Rivard, L. \& Cairney, . (2017). Relaive age effects in the Movement Assessment Battery for Children-2: age banding and scoring errors. Child Care Health and Development, 43(5), 752-757. doi:10.1111/ cch. 12459

Wang, J. H., Wu, M. C. \& Chang, H. H. (2013). Urban-rural disparity in physical fitness of elementary schoolchildren in Taiwan. Pediatrics International,55(3), 346- 354. doi:10.1111/ ped. 12044 